\title{
Single-walled carbon nanotube bundle under hydrostatic pressure studied by the first-principles calculations
}

\author{
Xiaoping Yand* \\ Group of Computational Condensed Matter Physics, \\ National Laboratory of Solid State Microstructures and Department of Physics, \\ Nanjing University, Nanjing 210093, P. R. China and \\ Department of Physics, Huainan Normal University, \\ Huainan, Anhui 232001, P. R. China \\ Gang Wu, Jian Zhou, and Jinming Dongt \\ Group of Computational Condensed Matter Physics, \\ National Laboratory of Solid State Microstructures and Department of Physics, \\ Nanjing University, Nanjing 210093, P. R. China
}

\begin{abstract}
The structural, electronic, optical and vibrational properties of the collapsed $(10,10)$ singlewalled carbon nanotube bundle under hydrostatic pressure have been studied by the first-principles calculations. Some features are observed in the present study: First, a collapsed structure is found, which is distinct from both of the herringbone and parallel structures obtained previously. Secondly, a pseudo-gap induced by the collapse appears along the symmetry axis $\Gamma X$. Thirdly, the relative orientation between the collapsed tubes has an important effect on their electronic, optical and vibrational properties, which provides an efficient experimental method to distinguish unambiguously three different collapsed structures.
\end{abstract}

PACS numbers: $61.46 .+w, 73.22 .-f, 78.67 . \mathrm{Ch}, 78.30 . \mathrm{Na}$ 


\section{Introduction}

In the past decade carbon nanotubes (CNTs) [1-3], both single-walled (SWNT) and multi-walled (MWNT), had been extensively investigated due to their special electrical and mechanical properties, as well as their potential applications in future nanostructured materials, such as nanoscale quantum wires, single electron and field-effect transistors and sensors.

It is well known that the physical properties of the CNTs depend much on their geometrical structures, and so can be easily changed by an applied pressure or strain, which could be used to fabricate the nanoscale electromechanical coupling devices and transducers. For example, a uniaxial strain on the SWNTs can cause a metal-semiconductor transition. On the other hand, both of the SWNT and MWNT bundles under hydrostatic pressure [4-15] have been studied experimentally and theoretically, showing a structural phase transition (SPT) at a critical pressure. It is found that the Raman peaks shift to higher frequencies with increasing hydrostatic pressure, and the radial breathing mode (RBM) disappears from the spectrum above the critical pressure [5,7]. Very recently, Elliott et al. [13] reported their classical molecular dynamics simulations on the SWNT bundles under hydrostatic pressure, which can collapse to a herringbone structure after the SPT, and their Raman spectrum measurement showed an excellent agreement with their simulations. However, Zhang et al. [16] put forward that the stress-induced herringbone structure is not the most favorable in energy, but found a parallel array of the collapsed nanotubes which is even more favorable. Therefore, it is unclear up to now which one, the parallel or the herringbone, is the most favorable structure of the SWNT bundles above the critical hydrostatic pressure.

It is well known that the optical spectroscopy, from the Raman and infrared modes to the visible optical absorption spectrum, is a powerful experimental method to detect the geometrical structures of the systems, because they are sensitive to the changes of the geometrical structures and electronic structure of the materials, especially in the nanostructured materials, such as CNTs. So, in this paper, we systematically investigate electronic, optical and vibrational properties of the different collapsed structures for the $(10,10)$ SWNT bundles under hydrostatic pressure using the first-principles method, in order to identify them unambiguously.

The paper is organized as follows: In Sec. II, we introduce the employed method. Then, the calculated results and discussions are given in Sec. III. Finally, some concluding remarks are offered in Sec. IV.

\section{Method}

We firstly study the changes of geometrical structure driven by a combination of hydrostatic pressure and van der Waals forces. The zero-temperature structural minimizations of the enthalpy $(H=U+P V)$ were carried out on a supercell containing a $2 \times 2 \times 2(10,10)$ SWNT bundle using the universal force field (UFF) method $[17,18]$. We only change the diagonal part of pressure tensor, which ensure the hydrostatic pressure is applied. In order to induce a structural phase transition, a step-wise monotonically increasing hydrostatic pressure was applied to the $(10,10)$ SWNT bundle at zero temperature. The pressure was increased from 0 to $5 \mathrm{GPa}$ in 50 steps, minimizing the enthalpy of the SWNT bundle after each pressure increment. The geometrical structures of the collapsed SWNT bundle were optimized again employing conjugate gradient technique through the first-principles method 
[19], and in the final geometry no forces on the atoms exceed $0.001 \mathrm{eV} / \AA$. However, no qualitative structure difference is found after the further first-principles geometry optimization. Then the total energy plane-wave potential method [19] in the framework of local density approximation (LDA) has been used to investigate the structural, electronic, optical and vibrational properties of the collapsed SWNT bundle under hydrostatic pressure. The ionelectron interaction was modeled by the projector augmented wave (PAW) potentials with a uniform energy cutoff of $500 \mathrm{eV}$. The maximum spacing between $\mathrm{K}$ points was $0.03 \AA^{-1}$ and the smearing width was taken to be $0.04 \mathrm{eV}$ in the ground state. The Raman intensity was calculated by combining the first-principles calculations and the empirical bond polarizability model (EBPM) $[20,21]$.

\section{Results and Discussions}

Taking the example of collapse to the parallel structure, the volume change was measured as a function of the applied hydrostatic pressure using the UFF method, given in Fig. 1(a), from which a discontinuous SPT can be clearly seen at about $3 \mathrm{GPa}$. In Fig. 1(b)-(d) shown are three different collapsed structures of the $(10,10)$ SWNT bundle after the SPT at $3 \mathrm{GPa}$, among which the parallel structure [Fig. 1(b)] and the herringbone structure [Fig. 1(d)] are in good accordance with the classic molecular dynamics simulation results, obtained previously in Ref.[16] and Ref.[13], respectively. It can be seen that the SWNTs spontaneously collapse to those with a dumbbell-like cross section, where the separation between two opposite parallel walls is approximately equal to the distance between layers in turbostratic graphite. However, we have also found an in-between configuration [Fig. 1(c)], which is an almost degenerate in enthalpy with the parallel one, having a difference of only $0.3 \mathrm{meV}$ per atom (see the following Table I). It should be noted that all tubes are equivalent to each other in a unit cell of the parallel structure, but are different in other two structures, in which there are two inequivalent tubes in their one unit cell. For comparison, we list in Table I all our LDA results of the three collapsed structures, from which we can find both of the parallel and in-between structures are more favorable in enthalpy than the herringbone one, but the largest difference of the enthalpy per atom in the three structures is only about $2.4 \mathrm{meV}$. So, it is very important to identify them clearly in future experiments.

The electronic band-structure along the nanotube symmetry axis $\Gamma X$, for an individual $(10,10)$ SWNT and its bundle at $0 \mathrm{GPa}$, and the three different collapsed structures at $3 \mathrm{GPa}$ are calculated by the LDA method and given in Figs. 2(a)-2(e), respectively. Here $k$-points sampling of $1 \times 1 \times 120$ is used in our calculations. The intertube van der Waals forces and the structure deformation break the rotational symmetry of the SWNT even at zero pressure, making its energy bands near Fermi Level split, as shown in Fig. 2(b), which is different from that of an individual SWNT [Fig. 2(a)] at the same zero pressure. Also, the density of states (DOS) for the three collapsed structures has also been calculated with a $k$-points sampling of $0.02 \AA^{-1}$ in the Brillouin Zone (BZ), and shown in Fig. 3. From a comparison between Fig. 2 and 3, it is found that although the three collapsed structures always remain in metal [as seen in Fig. 3], there exists still a basic difference between their band structures. For example, the band structure along the $\Gamma X$ direction [Fig. 2(c)] of the parallel structure shows a pseudo-gap of $0.2 \mathrm{eV}$ at the Fermi Level, and another small one of $0.08 \mathrm{eV}$ emerges in the electronic band of the herringbone structure [Fig. 2(e)]. However, no the pseudo-gap exists in the band of the in-between structure, in which the top of the valence band and the bottom of the conduction band lie separately at different $k$ points without the pseudo-gap in Fig. 2(d). In fact, this kind of band differences between the three collapsed structures 
comes from the different weak dispersions along the directions perpendicular to the tube axis, caused by the different numbers and relative orientation of the inequivalent tubes in their unit cells.

The changes of the electronic structure can be embodied well in their optical absorptions, which are calculated with the $k$-points sampling of $1 \times 1 \times 120$ for the individual SWNT and of $0.02 \AA^{-1}$ in the BZ for other bundle structures, and shown in Figs. 4(a)-4(e). The individual $(10,10)$ SWNT [Fig. 4(a)] has six absorption peaks polarized along tube direction (Z direction), lying at 1.591, 2.788, 3.516, 3.903, 11.92 and $14.069 \mathrm{eV}$, respectively, which are almost the same as those in Ref [22]. For the bundle at zero pressure, the original characteristic peaks of the individual SWNT shown in Fig. 4(a) are slightly shifted because its band splitting caused by the tube-tube interaction in the bundle, as seen clearly in Fig. 4(b). In addition, a strong absorption peak polarized along tube direction emerges at 0.184 $\mathrm{eV}$ due to the existing pseudo-gap along the direction parallel the $\Gamma X$, which had been predicted by Paul Delaney et al. [23] and further confirmed by a following experiment [24]. To investigate the evolution of this strong absorption peak and the original six ones with the applied hydrostatic pressure, we label them with symbols of ' 0 ', ' '1', '2', '3', '4', '5' and '6', respectively, in Fig. 4, and list their calculated energy positions in Table II.

It is seen from Fig. 4 and Table II that: 1) the original distinct peaks of No. '4' and '5' for the undeformed $(10,10)$ SWNT bundle in Fig. 4(b) become indistinct or disappear in Figs.4(c)-4(e) for three collapsed structures, and other characteristic peaks in Figs. 4(b) are blue-shifted or red-shifted. 2) The peak of No. '0' is blue-shifted from $0.184 \mathrm{eV}$ in Fig. 4(b) to $0.354 \mathrm{eV}$ in Fig, 4(c) for the parallel structure and to $0.53 \mathrm{eV}$ in Fig. 4(e) for the herringbone one. But, it makes a slightly red-shift to $0.15 \mathrm{eV}$ in Fig. 4(d) for the in-between structure. 3) The peak of No. ' 1 ' is red-shifted for the parallel and the in-between structures, as shown in Fig. 4(c) and 4(d), but splits into three distinctive peaks at 1.149, 1.584 and $1.965 \mathrm{eV}$ for the herringbone structure, respectively, seen in Fig. 4(e). It seems that all the above characteristics of the optical absorption, especially their lower frequency peaks, could be used to identify experimentally three different collapsed structures.

On the other hand, it can be found from Figs. 4(b)-4(e) that there exists a large difference in the optical absorption anisotropy for the undeformed $(10,10)$ SWNT bundle and other three collapsed configurations, which is listed as follows: 1) First of all, the optical responses polarized along $\mathrm{X}$ and $\mathrm{Y}$ directions are almost the same in magnitude in the undeformed and the herringbone structures, but much different in the parallel and the in-between ones, which could be caused by the relative orientations between the nearest tubes in them. 2) In addition, it can be found that the optical responses along $\mathrm{X}$ direction for both of the parallel and in-between ones are almost the same in magnitude too, and in the low-energy region weaker than that polarized along Y direction, because in both structures the normal of the flat segment of the collapsed tubes makes an almost the same angle with X or Y directions. The flat segment of the collapsed dumbbell-like cross section [see Fig. 1(b)] looks very similar to the graphite sheet. It is well known that in graphite, the optical absorption component polarized perpendicular to the carbon-layer is much smaller than that parallel to the layer [see Ref. 22]. It is seen clearly from Fig. 1(b) and 1(c) that the Y component of the optical absorption in both parallel and in-between structures has a more parallel component of the graphite than the X component, which is finally reflected in Fig. 4(c) and $4(\mathrm{~d})$. We have repeated the same calculations for other SWNT bundles with the larger radius [for example, the armchair $(12,12),(14,14),(16,16),(18,18)$ and $(20,20)$ tubes], and found the flat segments in their cross sections become the larger and larger with increasing 
tube radius, indicating the graphite-like characteristic anisotropy would be more notable in the collapsed SWNT bundles with larger radii. On the other hand, this character is absent in the optical spectra of the herringbone configuration because as seen from Fig. 1(d), its $\mathrm{X}$ and $\mathrm{Y}$ directions form an almost the same angle with the flat segment of the collapsed tubes, making so the $\mathrm{X}$ and $\mathrm{Y}$ components of its optical absorptions contain almost the same amount of the parallel component of the graphite.

Now, let us discuss their vibrational properties. It is known that the RBM is the most important low-frequency mode in the Raman spectra of a perfect SWNT, in which all carbon atoms are subject to an in-phase radial displacement. It was found that the RBM frequency of a SWNT is independent of its chiral angle and depends only on its diameter in an inverse proportion. Here, we have calculated by the EBPM the nonresonant Raman spectra of a (10 10) SWNT bundle at $0 \mathrm{GPa}$ and its three different collapsed structures at $3 \mathrm{GPa}$, and shown them in Fig. 5. Because there is no obvious difference between the Raman spectra of three collapsed structures in high-energy region, only the Raman peaks in the range from 0 to $350 \mathrm{~cm}^{-1}$ are given in Fig. 5. From Fig. 5(a) and its inset, we can find the RBM of the individual SWNT moves from $174 \mathrm{~cm}^{-1}$ to the higher energy $205 \mathrm{~cm}^{-1}$ in the undeformed bundle due to the van der Waals forces. Meantime, its low-frequency peak at about 100 $\mathrm{cm}^{-1}$ seen in the inset of Fig. 5 (a) splits, producing several peaks in Fig. 5(b). After the SPT, it is clearly seen from Figs. 5(b)-5(d) that the Raman spectra of three collapsed structures have been changed greatly: (1) the whole Raman spectra extend more widely than before the SPT; (2) more Raman peaks emerge, e.g., the big distinctive peak at about $300 \mathrm{~cm}^{-1}$; (3) Most importantly, the Raman peaks shift to higher frequencies. We made a search for the Raman frequency range from 180 to $350 \mathrm{~cm}^{-1}$ by detailed analysis on the vibration modes of the Raman peaks, but no RBM-like mode is found, i.e., the original RBM of the undeformed structure disappears from this frequency region, which is well consistent with the experimental results $[5,7]$.

All these changes in the Raman spectra are caused by the large structural difference before and after the SPT, leading to much different normal vibration modes after the SPT. Specifically, by detailed analysis on the vibration modes shown in Fig. 5(b) and 5(d), two main differences can be found in them: firstly a distinct Raman peak at $177 \mathrm{~cm}^{-1}$ (quadrupole vibration mode) in the parallel structure disappears from the corresponding spectrum range of the herringbone structure, in which, however, the quadrupole vibrations induce two strong Raman-active peaks at 115 and $136 \mathrm{~cm}^{-1}$ due to coupling with other vibration modes in low-energy region; and secondly some Raman peaks appear below $100 \mathrm{~cm}^{-1}$ in the herringbone structure, which should be mainly produced by the relative vibrations between two inequivalent tubes in its unit cell. In order to illuminate these vibration modes, we show the atomic displacements at $177 \mathrm{~cm}^{-1}$ for the parallel structure and 81,115 and $136 \mathrm{~cm}^{-1}$ for herringbone one in Figs. 6(a) and 6(c), respectively. The Raman peaks below $100 \mathrm{~cm}^{-1}$ also exist in that [Fig. 5(c)] of the in-between structure, but its Raman spectra above 100 $\mathrm{cm}^{-1}$ is very similar to that of the parallel structure with only a slight frequency shift, e.g. the quadrupole vibration mode at $177 \mathrm{~cm}^{-1}$ in Fig. 5(b) shifts to present $167 \mathrm{~cm}^{-1}$. For comparison, we also show its atomic displacements at 64 and $167 \mathrm{~cm}^{-1}$ in Fig. 6(b). So, our results indicate that the three collapsed structures can also be distinguished clearly by their low frequency Raman-active modes.

Experimentally, SWNT bundles contain tubes with different diameters or chiralities, forming the so-called "mixed" bundles in real sample, which seems to make difficult to compare the experimental data with our calculated results on the pure $(10,10)$ bundle be- 
cause unfortunately, at the present time, it is not possible to make a numerical simulation on the "mixed bundle" under hydrostatic pressure, using both classical mechanics and the first-principles method. Even so, however, our calculation results in this paper can still be helpful for analyzing the experimental data on the bundle.

In general, the Raman spectrum of the vibrational mode and x-ray diffraction of the tube bundles are often used to identify tubes' diameters and their chiral angles in the real sample. The X-ray analysis could reveal the average diameter of the tubes in the sample, and the Raman spectrum of the breathing mode of the bundle can also fix the diameter of the SWNTs in the bundle. Based upon all these data, we can identify rather accurately what kinds of SWNTs exist in the bundle by a comparison of the data with those of theoretical calculations. After that, we need to compare the experimental data, e.g., the SPT, the optical absorption and Raman spectrum, of the "mixed" SWNT bundle under pressure with those obtained from the theoretical calculations, which, of course, should be done for all possible pure SWNT bundle with different kinds of SWNTs in the real bundle sample. So, it would have no big problem for the experimentalist to relate their data directly to the theoretical analysis on the pure bundle structure. Usually, the $(10,10)$ tube is mostly grown in the real sample. That is why in this paper we made only calculation on the $(10,10)$ tube bundle.

\section{Summary}

In summary, we systematically investigate the structural, electronic, optical and Ramanactive properties of the collapsed $(10,10)$ SWNT bundle under hydrostatic pressure. We have found an in-between configuration under the collapse pressure, which is distinct from both the parallel and herringbone structures found previously, but is almost the degenerate in enthalpy with the parallel structure. In addition, we find the collapse possibly induces the pseudo-gap along tube axis $\Gamma X$, and the relative orientation between the collapsed tubes has an important effect on the electronic structure, optical and vibrational properties. Furthermore, our results indicate three different collapsed structures can be distinguished clearly by their electronic, optical and Raman-active properties.

\section{Acknowledgments}

This work was supported by the Natural Science Foundation of China under Grant No. 10474035 and A040108, and also by the State Key program of China through Grant No. $2004 \mathrm{CB} 619004$.

* Electronic address: bunnyxp@hotmail.com

$\dagger$ Corresponding author. Email address: jdong@nju.edu.cn

1 S. Iijima, Nature (London) 354, 56 (1991).

2 T.W. Ebbesen, P.M. Ajayan, Nature (London) 358, 220 (1992).

3 J. Kong, H.T. Soh, A.M. Cassell, C.F. Quate, H. Dai, Nature (London) 395, 878 (1998).

4 A.K. Sood, P.V. Teredesai, D.V.S. Muthu, R. Sen, A. Govindaraj, and C.N.R. Rao, Phys. Status Solidi (b) 215, 393 (1999). 
5 U.D. Venkateswaran, A.M. Rao, E. Richter, M. Menon, A. Rinzler, R.E. Smalley, and P.C. Eklund, Phys. Rev. B 59, 10928 (1999).

6 S. Reich, H. Jantoljak, and C. Thomsen, Phys. Rev. B 61, R13389 (2000).

7 M.J. Peters, L.E. McNeil, J.P. Lu, and D. Kahn, Phys. Rev. B 61, 5939 (2000).

8 P.V. Teredesai, A.K. Sood, D.V.S. Muthu, R. Sen, A. Govindarai, and C.N.R. Rao, Chem. Phys. Lett. 319, 296 (2000).

9 U.D. Venkateswaran, E.A. Brandsen, U. Schlecht, A.M. Rao, E. Richter, I. Loa, K. Syassen, and P.C. Eklund, Phys. Status Solidi (b) 223, 225 (2001).

10 J. Tang, L.-C. Qin, T. Sasaki, M. Yudasaka, A. Matsushita, and S. Iijima, Phys. Rev. Lett. 85, 1887 (2000).

11 S.M. Sharma, S. Karmakar, S.K. Sikka, P.V. Teredesai, A.K. Sood, A. Govindaraj, and C.N.R. Rao, Phys. Rev. B 63, 205417 (2001).

12 S. Rols, I.N. Gontcharenko, R. Almairac, J.L. Sauvajol, and I. Mirebeau, Phys. Rev. B 64, 153401 (2001).

13 J.A. Elliott, J.K.W. Sandler, A.H. Windle, R.J. Young, and M.S.P. Shaffer, Phys. Rev. Lett. 92, 095501 (2004).

14 N.G. Chopra, L.X. Benedict, V.H. Crespi, M.L. Cohen, S.G. Louie and A. Zettl, Nature (London) 377, 135 (1995).

15 M.H.F. Sluiter, and Y. Kawazoe, Phys. Rev. B 69, 224111 (2004).

16 X.H. Zhang, Z.F. Liu, and X.G. Gong, Phys. Rev. Lett. 93, 149601 (2004).

17 A.K. Rappe, C.J. Casewit, K.S. Colwell, W.A. Goddard, W.M. Skiff, J. Am. Chem. Soc. 114, 10024 (1992).

18 N. Yao, V. Lordi, J. Appl. Phys. 84, 1939 (1998).

19 G. Kresse, J. Hafner, Phys. Rev. B 47, R558 (1993); ibid 49, 14251 (1994); G. Kresse, J. Furhmuller, Software VASP, Vienna (1999); Phys. Rev. B 54, 11169 (1996); Comput. Mat. Science 6, 15 (1996).

20 S. Guha, J. Menendez, J.B. Page, and G.B. Adams, Phys. Rev. B 53, 13106 (1996); R. Saito, T. Takeya, T. Kimura, G. Dresselhaus, and M.S. Dresselhaus, Phys. Rev. B 57, 4145 (1998).

21 Gang Wu, Jian Zhou and Jinming Dong, Phys. Rev. B 72, 115411 (2005).

22 G.Y. Guo, K.C. Chu, Ding-sheng Wang, Chun-gang Duan, Phys. Rev. B 69, 205416 (1996).

23 Paul Delaney, Hyoung Joon Choi, Jisoon Ihm, Steven G. Louie and Marvin L. Cohen, Nature 391, 466 (1998); Phys. Rev. B 60, 7899 (1999).

24 Min Ouyang, Jin-Lin Huang, Chin Li Cheung, Charles M. Lieber, Science 292, 702 (2001). 


\section{TABLE}

Table I. Total energies (E) and enthalpies $(\mathrm{H})$ of the parallel, the new in-between, and the herringbone structures at the collapsed pressure of $3 \mathrm{GPa}$ and zero temperature.

\begin{tabular}{|l|l|l|l|}
\hline Stucture & E/atom $(\mathrm{eV})$ & PV/aotm $(\mathrm{eV})$ & $\mathrm{H} / \operatorname{aotm}(\mathrm{eV})$ \\
\hline Parallel & -9.9804 & 0.1639 & -9.8165 \\
\hline New in-between & -9.9809 & 0.1647 & -9.8162 \\
\hline Herringbone & -9.9839 & 0.1698 & -9.8141 \\
\hline
\end{tabular}

Table II. The calculated energy positions of the characteristic optical absorption peaks [labelled by symbol of '0', '1', '2', '3', '4', '5' and '6'] polarized parallel to the tube axis (Z direction) in Fig. 4(a)-4(e).

\begin{tabular}{|llllllll|}
\hline character peak & 0 & 1 & 2 & 3 & 4 & 5 & 6 \\
\hline Stucture & & 1.591 & \multicolumn{2}{l}{ Energy $(\mathrm{eV})$} \\
\hline Individual $(0 \mathrm{GPa})$ & & 2.788 & 3.516 & 3.903 & 11.920 & 14.069 \\
\hline Bundle $(0 \mathrm{GPa})$ & 0.184 & 1.544 & 2.836 & 3.311 & 3.869 & 11.941 & 14.022 \\
\hline Parallel $(3 \mathrm{GPa})$ & 0.354 & 1.367 & 2.434 & 3.318 & 4.155 & 12.097 & 14.423 \\
\hline New in-between $(3 \mathrm{GPa})$ & 0.150 & 1.516 & 2.768 & 3.291 & 3.992 & 12.009 & 14.443 \\
\hline Herringbone $(3 \mathrm{GPa})$ & 0.53 & $1.149,1.584,1.965$ & 2.904 & 3.339 & 3.788 & 12.118 & 14.409 \\
\hline
\end{tabular}




\section{Figure Captions}

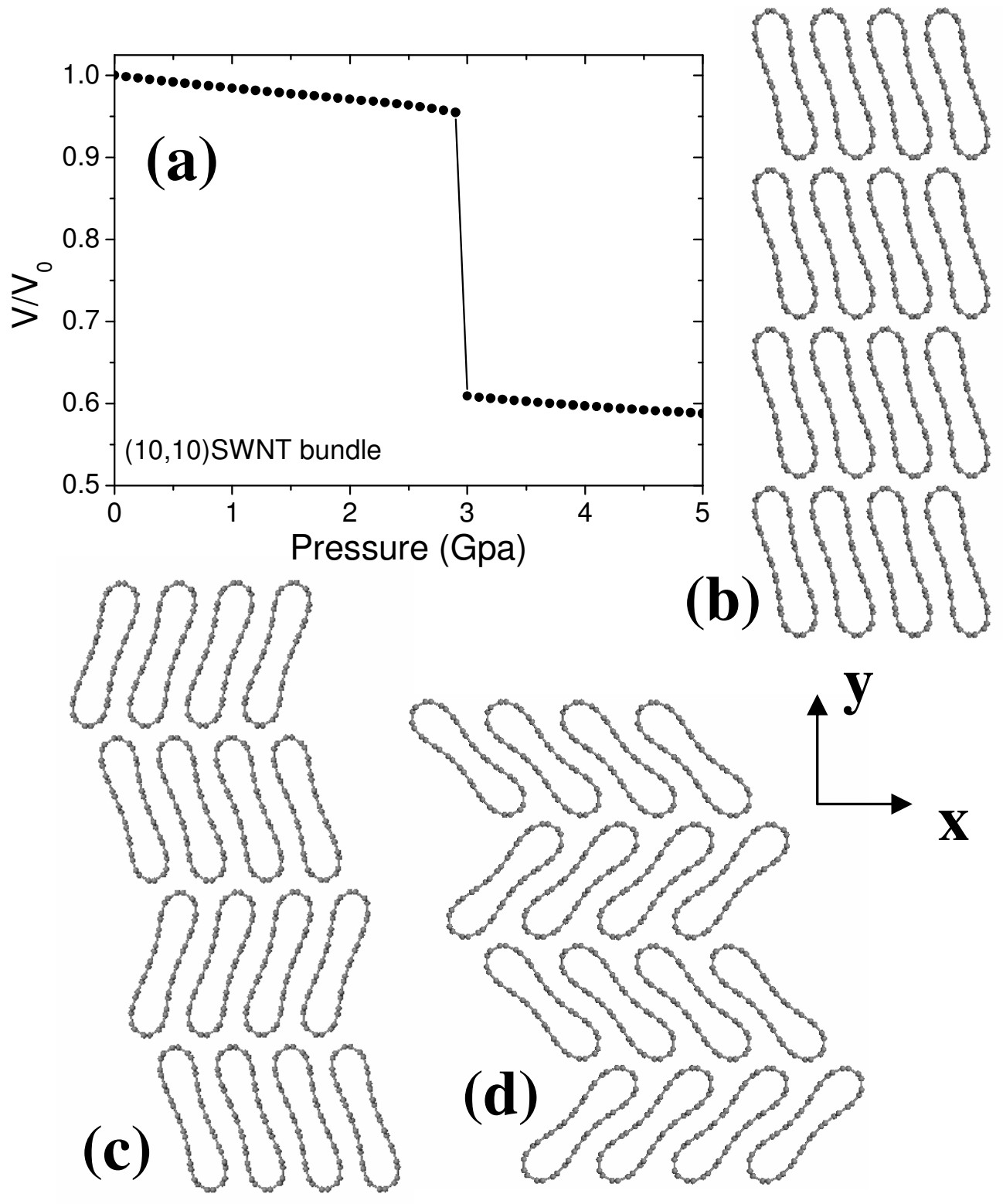

FIG. 1: Color online)(a) Loading curve for $(10,10)$ SWNT bundle as a function of hydrostatic pressure. Snapshots of the cross section of a $(10,10)$ SWNT bundle at 3 GPa: (b) the parallel structure, (c) the in-between structure, and (d) the herringbone structure. 


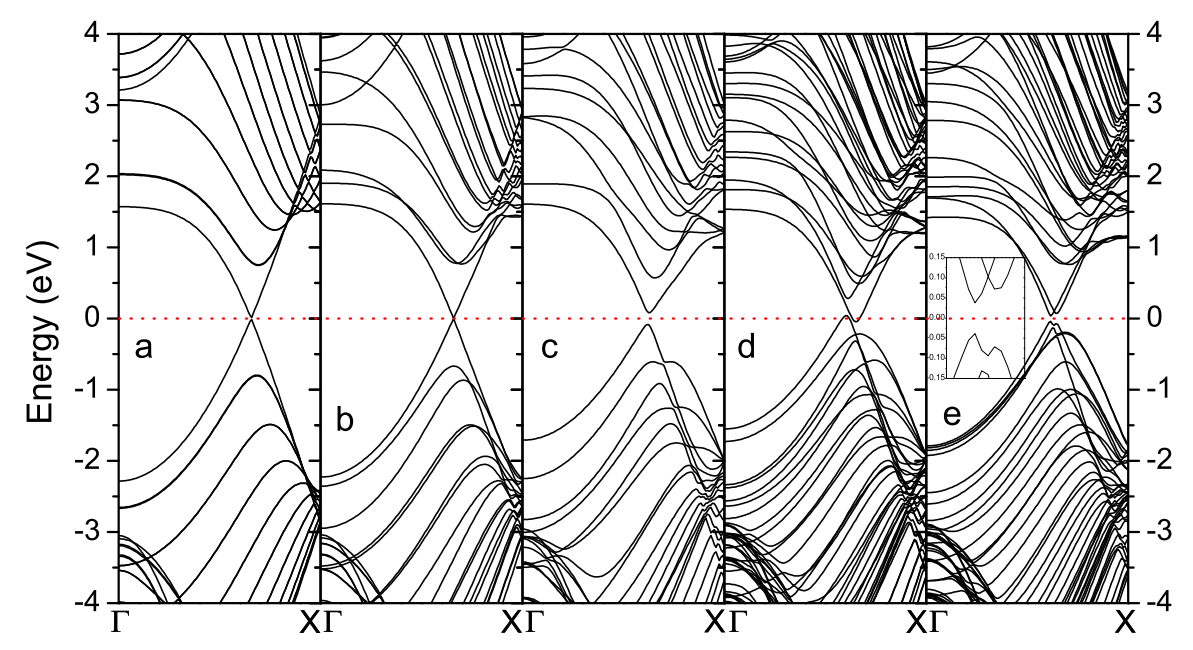

FIG. 2: (Color online) The calculated electronic band structures along the nanotube symmetry axis $\Gamma X$ of (a) an individual $(10,10)$ SWNT at $0 \mathrm{GPa}$, (b) a $(10,10)$ SWNT bundle at 0 GPa, and of three collapsed structures at $3 \mathrm{GPa}$ (c) the parallel structure, (d) the in-between structure, (e) the herringbone structure. Inset of (e) shows the pseudo-gap at the Fermi level in an energy region of $-0.15 \sim 0.15 \mathrm{eV}$. The Fermi level is set at zero. 


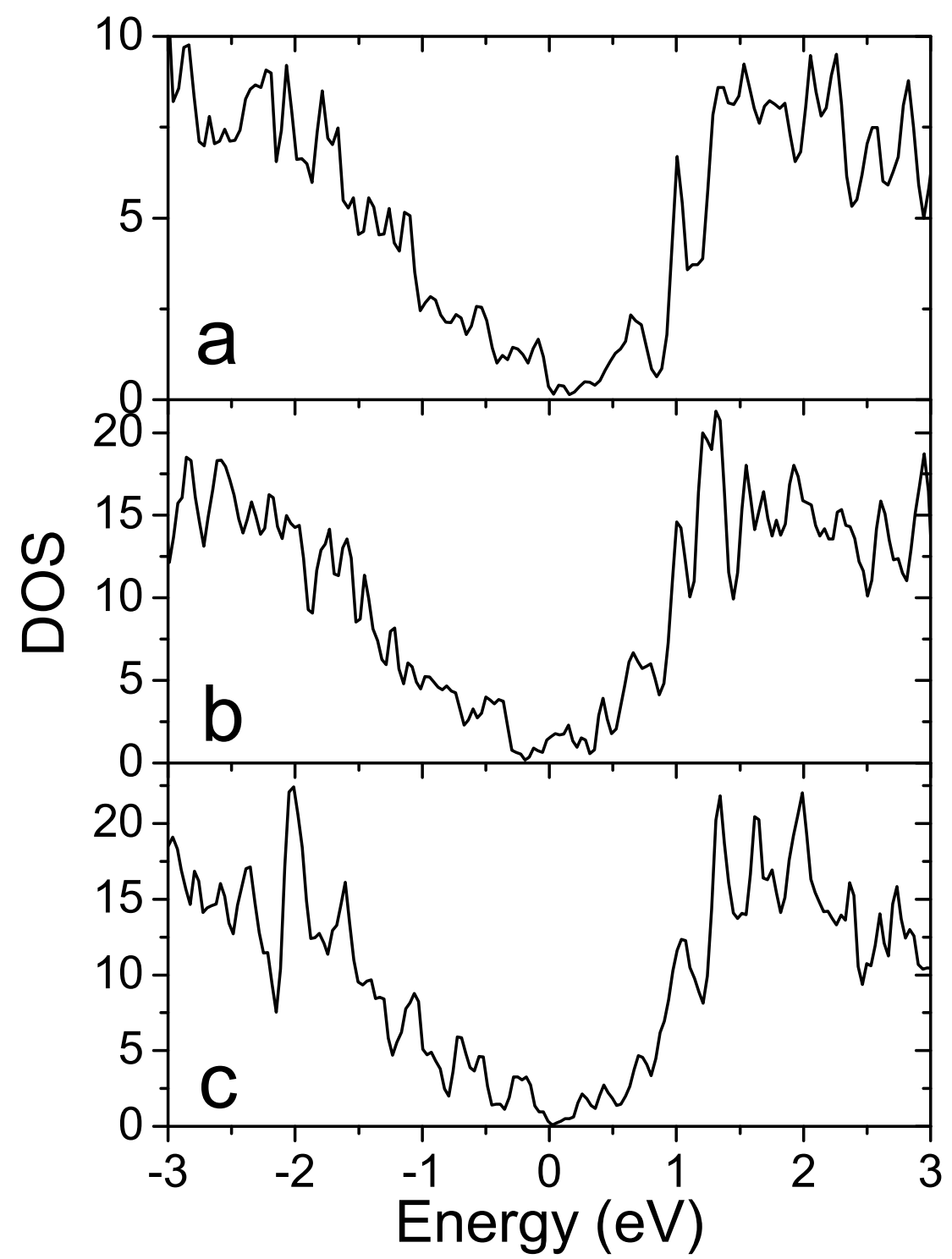

FIG. 3: The calculated density of states for three collapsed structures at 3 GPa: (a) the parallel structure, (b) the in-between structure, and (c) the herringbone structure. The Fermi level is set at zero. 


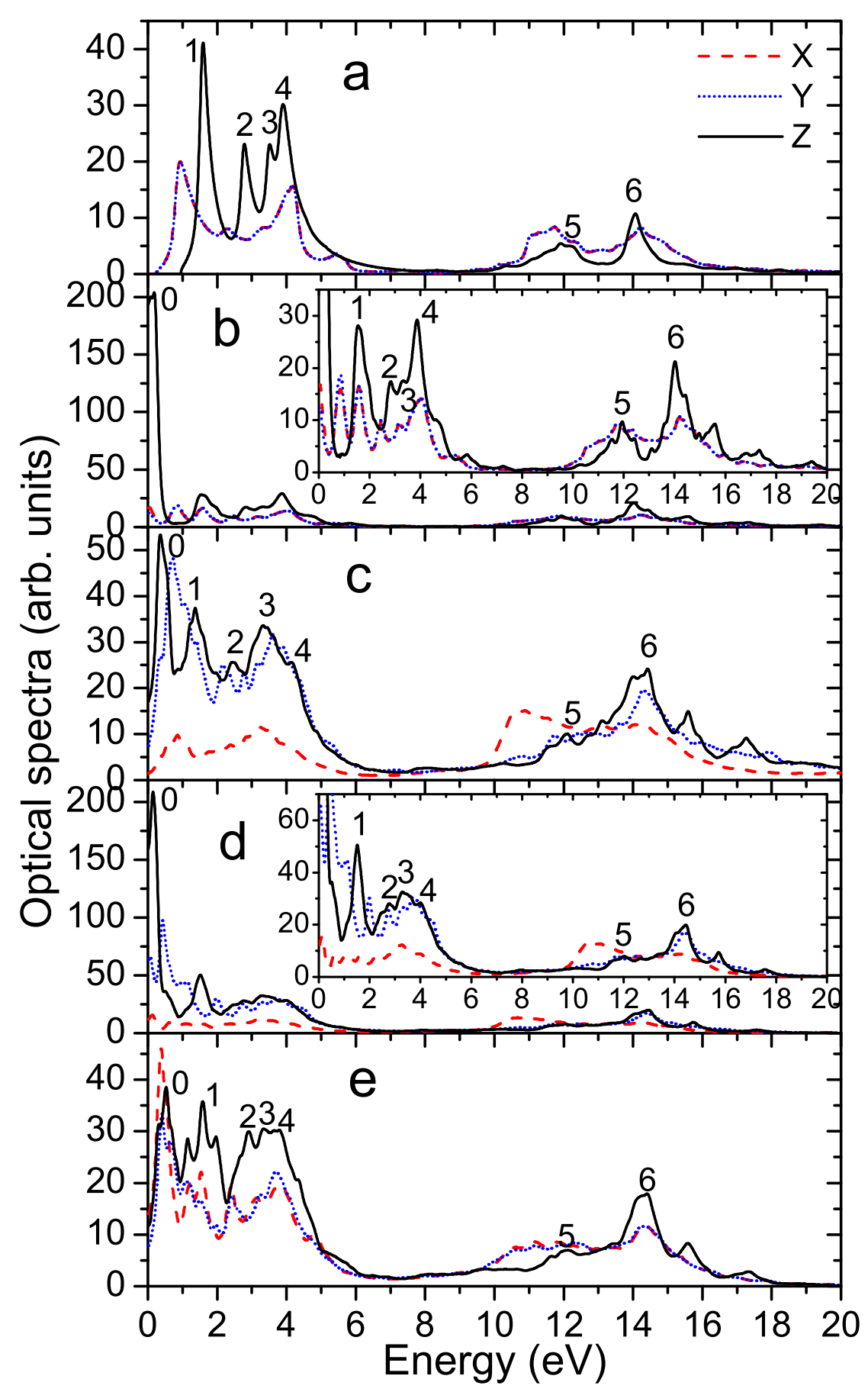

FIG. 4: (Color online) The calculated imaginary part (absorptive part) of dielectric function polarized along X, Y and Z directions, respectively, for (a) an individual $(10,10)$ SWNT at 0 GPa, (b) a $(10,10)$ SWNT bundle at $0 \mathrm{GPa}$, and for three collapsed structures at $3 \mathrm{GPa}$ : (c) the parallel structure, (d) the in-between structure, and (e) the herringbone structure. 


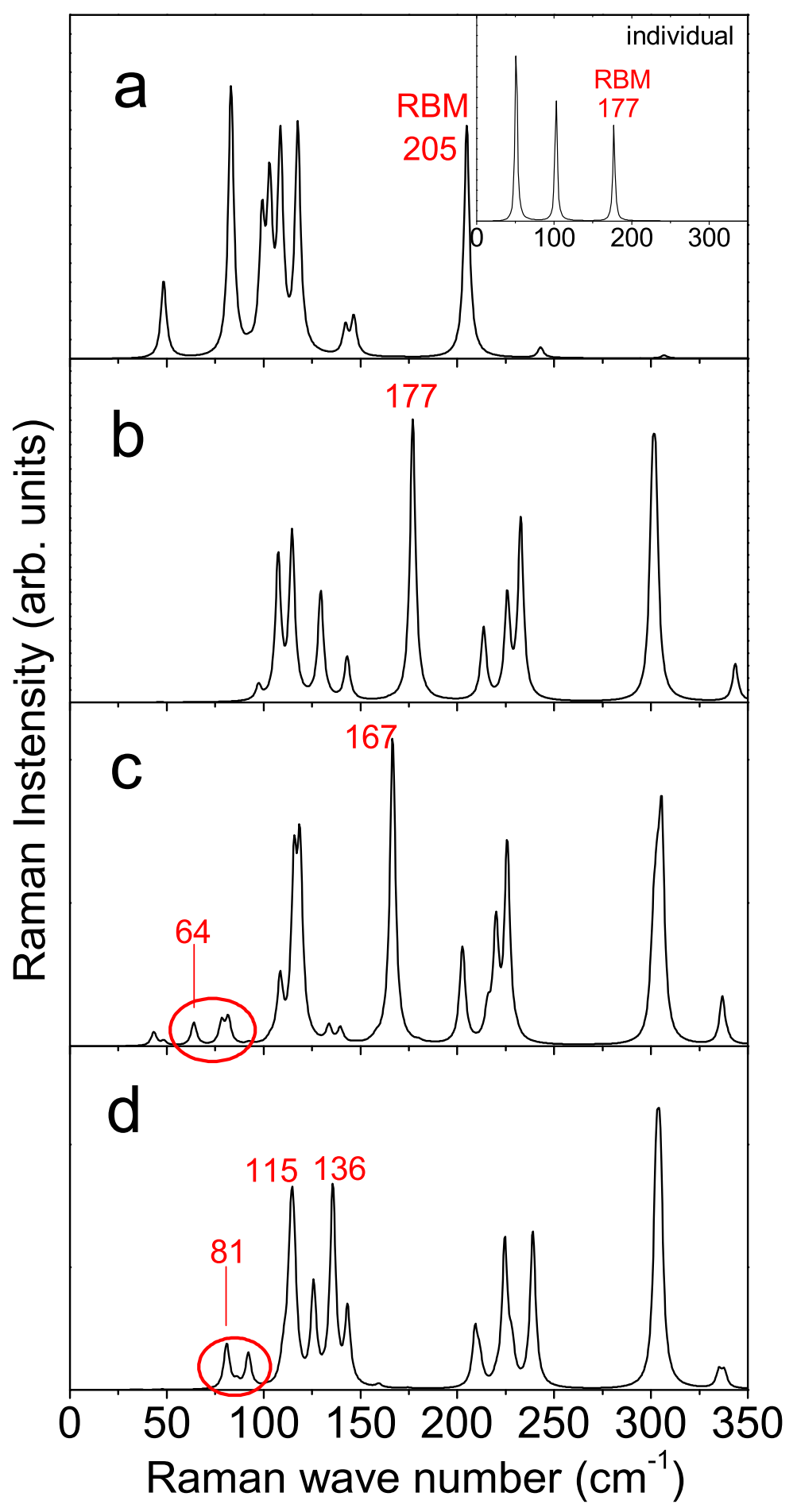

FIG. 5: (Color online) The calculated nonresonant Raman spectra of (a) a $(10,10)$ SWNT bundle at $0 \mathrm{GPa}$ (its inset shows that of an individual $(10,10) \mathrm{SWNT}$ ), and of three collapsed structures at 3 GPa: (b) the parallel structure, (c) the in-between structure, and (d) the herringbone structure. 

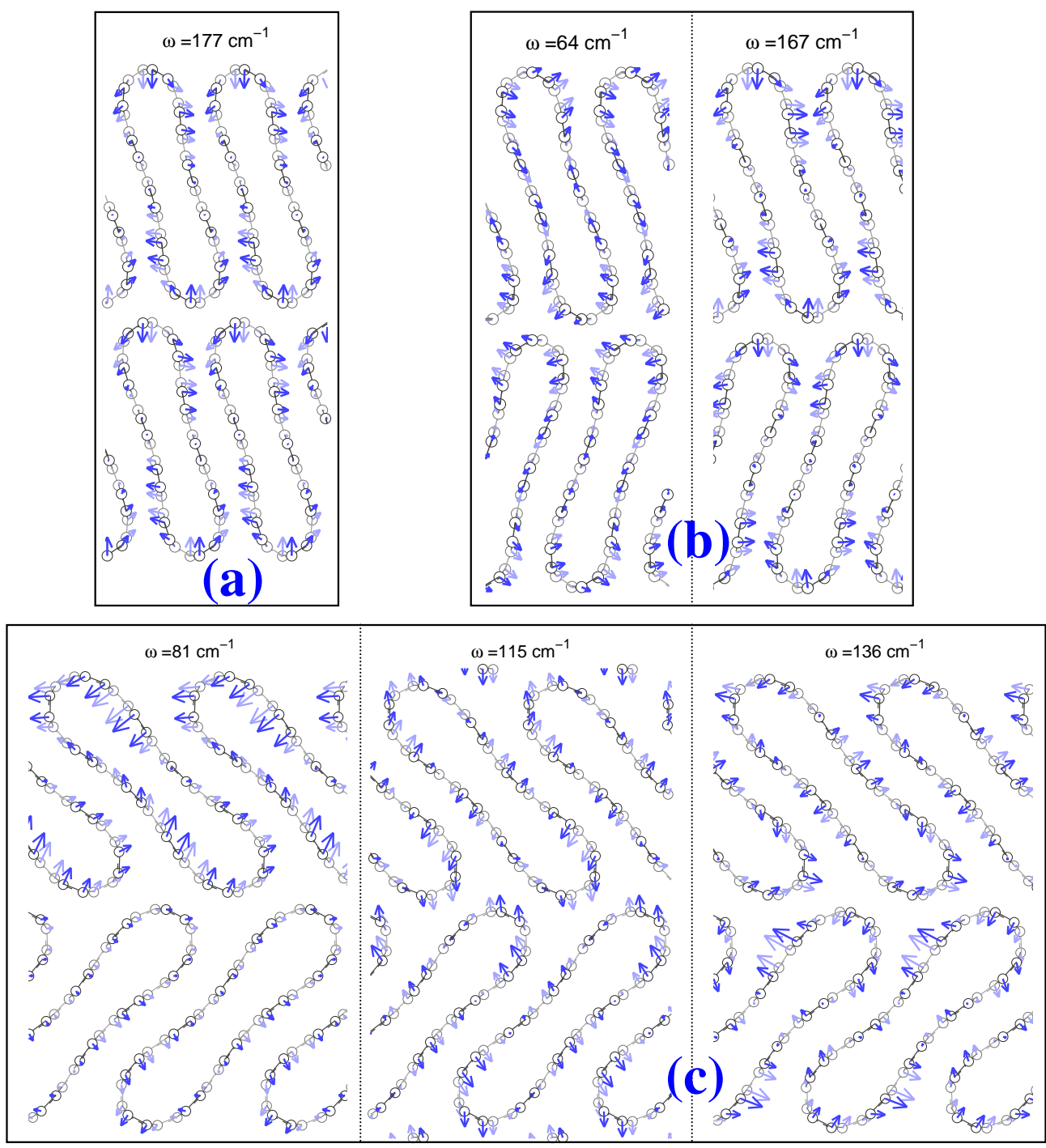

FIG. 6: (Color online) The eigenfrequencies and eigenvectors of some raman-active vibration modes for the different collapsed structures at $3 \mathrm{Gpa}$ : (a) the parallel structure, (b) the in-between structure, and (c) the herringbone structure. The small circles represent the carbon atoms and the straight lines between them indicate the bonds. The arrows represent the atomic motions. Deeper color arrow means the corresponding atom is nearer to the reader. 